\title{
Competition and interaction of polydisperse bubbles in polymer foams
}

\author{
S L Everitt, O G Harlen *, and H J Wilson ${ }^{1}$ \\ Department of Applied Mathematics, University of Leeds, Leeds LS2 9JT, UK
}

\begin{abstract}
The effects of interactions between bubbles of different sizes during bubble growth in a polymeric foam are investigated. Two models are used: a two-dimensional simulation in which both the effects of gas diffusion through the polymer and bubble interactions through fluid stresses are included, and a three-dimensional model in which bubbles are assumed to interact only through direct competition for gas, and diffusion of gas into the bubbles is instantaneous.

In the two-dimensional model, two different bubble sizes are used in a hexagonal array. For slow gas diffusion, the additional polymer stresses have little effect on the final bubble size distribution. For faster gas diffusion the growth occurs in two phases, just as was found in earlier work for isolated bubbles: an initial rapid viscous phase and a later phase controlled by the rate of polymer relaxation. In this later phase, polymers in the windows between neighbouring bubbles become highly stretched and these regions of high stress determine the dynamics of the growth.

In the three-dimensional model we consider the effects of rheology on a pair of different-sized spherical bubbles, interacting only through competition for available gas. Viscoelastic effects result in a wider distribution of bubble volumes than would be found for a Newtonian fluid.
\end{abstract}

Key words: Polymeric fluid; bubble growth; foam; bubble interactions; size distribution

\footnotetext{
* To whom correspondence should be addressed.

Email address: oliver@maths.leeds.ac.uk (O G Harlen).

1 Current address: Department of Mathematics, University College London, Gower Street, London WC1E 6BT, UK
} 


\section{Introduction}

Polymer foams are used in a wide variety of industrial applications, from car parts to insulation. One major method of manufacture is injection moulding, in which thermoplastic foam products are produced by injecting polymer that has been pressurised to enable it to absorb a blowing agent, and heated to a temperature above the glass transition temperature to allow moulding. Foaming results from a sudden reduction in pressure. After foaming is complete, the part is cooled at which point the polymer solidifies.

In this process, the product will be defective if a single large bubble spans a very large region of the mould, or if there is a significant variation in the foam density in different regions of the mould. For this reason, it is necessary to investigate the effect of various physical parameters on the size distribution of bubbles.

Much of the previous work in this area, including ours, has used the cell model to investigate individual gas bubbles, spherical because of their isolation, growing in either a Newtonian $[1,4,3,16,17,7]$ or viscoelastic $[19,20,21,8,2,13$, $12,6]$ fluid. A few authors $[15,11]$ have investigated more realistic geometries using Newtonian fluid models.

In Everitt, Harlen \& Wilson [5], we investigated the expansion of foams consisting of identical gas bubbles. Using a two-dimensional model with hexagonal cells we were able to ascertain how the presence of neighbouring bubbles affects the expansion of the foam and the stresses in the polymeric fluid. However, because the bubbles were identical this work provided no insight into the effects of competition between bubbles for a limited supply of gas.

Solving the coupled problem of gas diffusion and bubble expansion in a random distribution of bubbles in three dimensions is very difficult and so we consider two simplified models. In the first model we extend the two-dimensional, hexagonal, arrangement of bubbles studied in [5] to bubbles of two different sizes in order to investigate the effects of viscoelasticity and gas diffusion when bubbles are in close proximity to one another. In the second we consider a three-dimensional system in which two spherical bubbles of different sizes expand in a fluid in which the gas diffusion is effectively instantaneous. We examine the effect of viscoelasticity on the evolution of the bubble volume ratio. 


\section{Governing Equations}

The flow of an incompressible polymeric fluid with velocity field $\boldsymbol{u}$ may be described by the equations of conservation of mass:

$$
\nabla \cdot \boldsymbol{u}=0
$$

and momentum, in the absence of inertia:

$$
0=-\nabla p+\nabla \cdot \boldsymbol{\sigma}
$$

where $p$ is the pressure within the fluid, and $\boldsymbol{\sigma}$ is the fluid extra stress tensor, determined as a function of the flow history by the constitutive equation.

We assume that the expansion rate is sufficiently small that inertia may be neglected. This is justified provided the Reynolds number is sufficiently small, and an analysis using physical parameters for the growth of an isolated bubble was given in [6], predicting a Reynolds number of around $10^{-13}$. In a disordered array of bubbles, rapid rearrangement events may result in fast motion for which inertia is not negligible; we will not consider such events here.

We will model our fluid as an Oldroyd-B fluid, for which the stress is given by

$$
\begin{gathered}
\boldsymbol{\sigma}=2 \mu \boldsymbol{E}+G(\boldsymbol{A}-\boldsymbol{I}), \\
\boldsymbol{E}=\frac{1}{2}\left(\nabla \boldsymbol{u}+(\nabla \boldsymbol{u})^{T}\right), \\
\stackrel{\nabla}{\boldsymbol{A}}=-\frac{1}{\tau}(\boldsymbol{A}-\boldsymbol{I}),
\end{gathered}
$$

where

$$
\stackrel{\nabla}{\boldsymbol{A}}=\frac{\partial \boldsymbol{A}}{\partial t}+\boldsymbol{u} \cdot \nabla \boldsymbol{A}-\boldsymbol{A} \cdot \nabla \boldsymbol{u}-(\nabla \boldsymbol{u})^{T} \cdot \boldsymbol{A}
$$

is the upper convected derivative of the orientation tensor.

On the boundary between the fluid and the bubble, a stress balance normal to the surface (using normal vector $\boldsymbol{n}$ ) gives the boundary condition on $\boldsymbol{\sigma}$ :

$$
-p \boldsymbol{n}+\boldsymbol{\sigma} \cdot \boldsymbol{n}=-p_{g} \boldsymbol{n}+S \boldsymbol{n} \nabla \cdot \boldsymbol{n},
$$

where $S$ is the surface tension coefficient and $p_{g}$ the gas pressure in the bubble.

We model a situation analogous to injection moulding, in which gas is dissolved in the polymer solution and can diffuse into the bubbles. The expansion of bubbles in our fluid will be driven by an excess of gas, which is present in the fluid with a concentration $c(\boldsymbol{x}, t)$. The gas diffuses through the liquid obeying the advection-diffusion equation:

$$
\frac{D c}{D t}=\mathcal{D} \nabla^{2} c
$$


in which $\mathcal{D}$ is the diffusion coefficient.

For a bubble of total volume $V(t)$, the mass conservation equation governing the quantity $n$ of gas in this bubble is

$$
\frac{\mathrm{d} n}{\mathrm{~d} t}=\mathcal{D} \int_{\text {bubble surface }} \nabla c \cdot \boldsymbol{n} \mathrm{d} s
$$

and if we incorporate the gas law, this gives us an equation governing the pressure within the bubble:

$$
\frac{\mathrm{d}\left(p_{g} V\right)}{\mathrm{d} t}=R T \mathcal{D} \int_{\text {bubble surface }} \nabla c \cdot \boldsymbol{n} \mathrm{d} s
$$

in which $R$ is the gas constant and $T$ the temperature.

On the surface of each bubble, Henry's law governing the thermodynamics of the bubble interface states that

$$
c=H p_{g}
$$

where $H$ is the Henry's law constant. This constant controls the concentration of gas in the liquid at the bubble-liquid interface for a given bubble gas pressure. Henry's law is valid for dilute solutions where the gas concentrations are below $1 \%$. The typical values of $H$ and $p_{g 0}$, the initial gas pressure in the bubble, that we shall use, give an initial concentration of $0.46 \%$ (assuming a liquid density of around $1000 \mathrm{~kg} \mathrm{~mol}^{-1}$ and the molar mass of the gas as $0.044 \mathrm{~kg} \mathrm{~m}^{-3}$, corresponding to carbon dioxide).

\subsection{Dimensionless form of the equations}

We shall consider systems in which the gas concentration in the liquid is initially uniform. Henry's law (10) then forces the gas pressure in all the bubbles in the system to be instantaneously equal, with a value which we denote $p_{g 0}$. We use this value to make the gas concentration in the fluid dimensionless by scaling with $p_{g 0} / R T$. The system is not in equilibrium at time $t=0$ as the bubble pressure does not balance the surface tension, causing the bubbles and hence the system to expand against atmospheric pressure, $p_{a}$ at infinity. In all our simulations $p_{g 0}$ is chosen to be larger than the surface tension of the smaller bubbles so that both bubbles expand.

We make all times dimensionless with the fluid relaxation time, $\tau$, and lengths dimensionless with $R_{0}$, the initial radius of the largest bubble.

We scale all pressures (in both liquid and gas) using the difference from $p_{a}$, atmospheric pressure, relative to the initial difference $p_{g 0}-p_{a}$ between the bub- 
ble gas pressure and the external pressure, so that the dimensionless pressure variables are

$$
P=\frac{\left(p-p_{a}\right)}{\left(p_{g 0}-p_{a}\right)}, \quad P_{g}=\frac{\left(p_{g}-p_{a}\right)}{\left(p_{g 0}-p_{a}\right)} .
$$

The non-dimensionalisation used here is the same as in reference [5].

Our governing equations, with all quantities dimensionless, become:

$$
\begin{gathered}
\nabla \cdot \boldsymbol{u}=0, \\
0=-D e \nabla P+\nabla \cdot[2(1-\beta) \boldsymbol{E}+\beta(\boldsymbol{A}-\boldsymbol{I})], \\
\nabla=-(\boldsymbol{A}-\boldsymbol{I}), \\
\frac{D c}{D t}=N \nabla^{2} c,
\end{gathered}
$$

and

$$
\frac{\mathrm{d}}{\mathrm{d} t}\left(\frac{\left(p_{a}+\left(p_{g 0}-p_{a}\right) P_{g}\right) V}{p_{g 0}}\right)=N \int_{\text {bubble surface }} \nabla c \cdot \boldsymbol{n} \mathrm{d} s .
$$

We have introduced three dimensionless quantities: the Deborah number, De, the viscosity ratio $\beta$ and the dimensionless diffusivity $N$. These are defined

\begin{tabular}{|c|c|c|}
\hline$D e=\frac{\left(p_{g 0}-p_{a}\right) \tau}{(\mu+G \tau)}$ & $\begin{array}{l}\text { Deborah number: Ratio of the bubble growth rate } \\
\text { at the zero-shear-rate viscosity to the polymer re- } \\
\text { laxation rate }\end{array}$ & $1.8-9$ \\
\hline$\beta=\frac{G \tau}{(\mu+G \tau)}$ & $\begin{array}{l}\text { Viscosity ratio: Proportion of zero-shear-rate vis- } \\
\text { cosity contributed by polymer }\end{array}$ & $0-0.8$ \\
\hline$N=\frac{\mathcal{D} \tau}{R_{0}^{2}}$ & $\begin{array}{l}\text { Timescale ratio: Ratio of the polymer relaxation } \\
\text { time to a typical gas diffusion time }\end{array}$ & $0.1-\infty$ \\
\hline$\Gamma=\frac{R_{0}(\mu+G \tau)}{S \tau}$ & $\begin{array}{l}\text { Capillary number: ratio of viscous force (based on } \\
\text { zero-shear-rate viscosity) to surface tension }\end{array}$ & $\infty$ \\
\hline$\Phi=R T H$ & Dimensionless solubility of the gas in the liquid & 0.32 \\
\hline
\end{tabular}
fully in table 1 , along with the values these groups take in the results presented here; in table 2 we present the values we have used for the physical parameters from which they are derived.

Table 1

Dimensionless groups arising in the dimensionless equations of section 2.1

The boundary conditions at the liquid-bubble interface become:

$$
-D e P \boldsymbol{n}+[2(1-\beta) \boldsymbol{E}+\beta(\boldsymbol{A}-\boldsymbol{I})] \cdot \boldsymbol{n}=-D e P_{g} \boldsymbol{n}+\frac{1}{\Gamma} \boldsymbol{n} \nabla \cdot \boldsymbol{n}
$$

and

$$
c=\Phi \frac{\left(p_{a}+\left(p_{g 0}-p_{a}\right) P_{g}\right)}{p_{g 0}} .
$$


Table 2

Physical parameters

\begin{tabular}{lll}
\hline Parameter & Value & Units \\
\hline Gas constant, $R$ & 8.3 & $\mathrm{~J} \mathrm{~mol}^{-1} \mathrm{~K}^{-1}$ \\
Temperature, $T$ & 370 & $\mathrm{~K}$ \\
Henry's law constant, $H$ & 10.5 & $10^{-5} \mathrm{~mol} \mathrm{~N}^{-1} \mathrm{~m}^{-1}$ \\
Elastic modulus, $G$ & $0-10$ & $10^{5} \mathrm{~N} \mathrm{~m}^{-2}$ \\
Polymer relaxation time, $\tau$ & 1 & $\mathrm{~s}$ \\
Solvent viscosity, $\mu$ & $0.2-1$ & $10^{5} \mathrm{~N} \mathrm{~s} \mathrm{~m}^{-2}$ \\
Diffusivity, $\mathcal{D}$ & $0.1-\infty$ & $10^{-12} \mathrm{~m}^{2} \mathrm{~s}^{-1}$ \\
Initial radius of large bubble, $R_{0}$ & 1 & $10^{-6} \mathrm{~m}^{-1}$ \\
Surface tension coefficient, $S$ & 0 & $\mathrm{~N} \mathrm{~m}^{-1}$ \\
Pressure outside the fluid, $p_{a}$ & 1 & $10^{5} \mathrm{~N} \mathrm{~m}^{-2}$ \\
Initial bubble gas pressure, $p_{g 0}$ & 10 & $10^{5} \mathrm{~N} \mathrm{~m}^{-2}$ \\
\hline
\end{tabular}

Two further dimensionless variables have been introduced here: $\Gamma$, the capillary number, and $\Phi$, which is a dimensionless representation of the solubility of the gas in the liquid. These are also defined in table 1.

\section{Bidisperse hexagonal array of bubbles}

In reference [5] we investigated the growth of identical bubbles in a twodimensional hexagonal array. One of the principal observations of this work was that, at high Deborah number, the large elastic stresses generated in the edges between bubbles control the second stage of bubble growth, where the bubble pressure is balanced by the elastic stress. However, since all the bubbles were identical, symmetry arguments guaranteed that each bubble would absorb exactly its share of the available gas, so the only effect of competition between bubbles for limited gas supply was a global reduction in growth rate as the gas supply ran down.

In this paper, we extend the two-dimensional simulation method to consider bubbles of different sizes. In the earlier work, a periodic unit containing 1/12 of a single bubble was sufficient. Here, by effectively tripling the size of our computational domain, we can extend the scope to systems in which there are two different initial bubble sizes, one occurring twice as frequently as the other - see figure 1. Throughout this section, we will refer to the less frequently occurring bubble as bubble 1 and the more frequently occurring 
bubble as bubble 2 . In cases where the initial sizes of bubbles 1 and 2 are equal we reproduce the results of the simulations in reference [5].

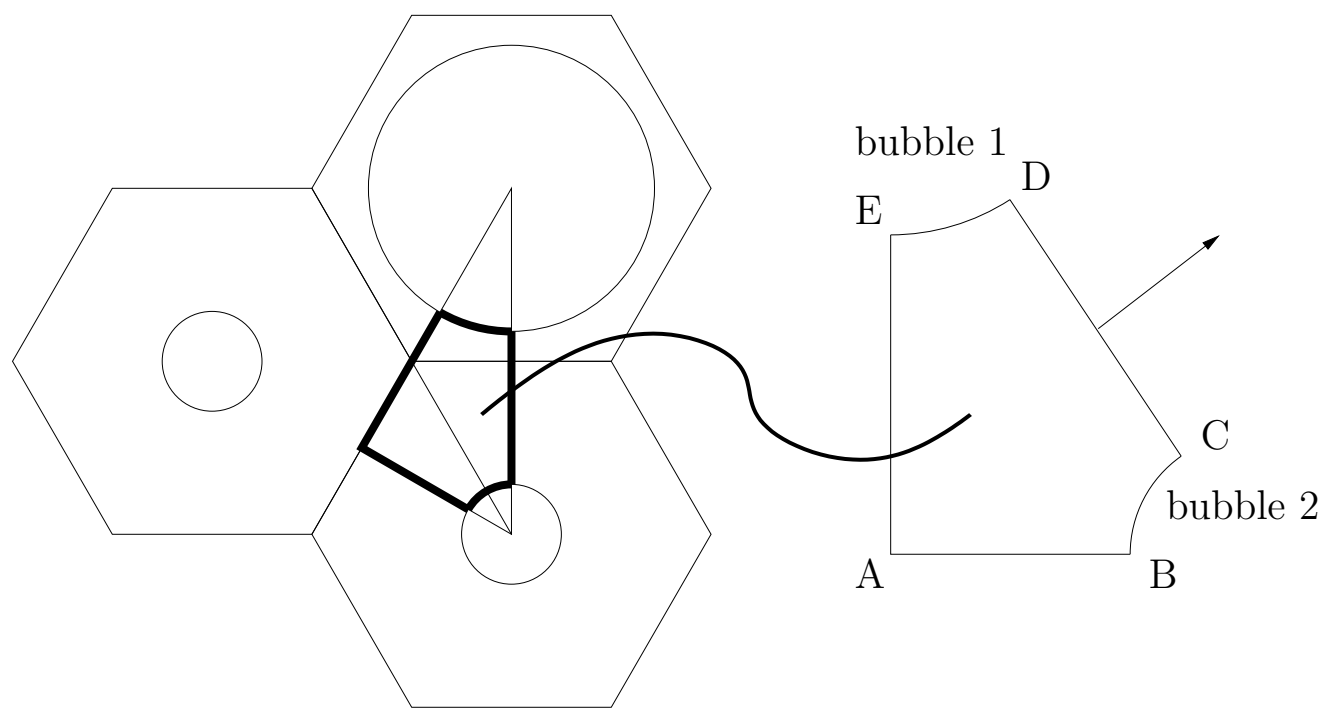

Fig. 1. Diagram showing the two dimensional arrangement of bubbles.

\subsection{Governing Equations and Boundary Conditions}

We assume that the bubbles are initially circular with a uniform initial gas concentration profile in the liquid so that the initial gas pressures in the bubbles are equal. We also assume that the polymers in the liquid are unstretched at $t=0$. In a foaming process bubbles nucleate at different times producing bubbles of different sizes. Nucleation is a highly complex process and has been the subject of much investigation $[10,14,18]$. Here, we do not attempt to model the nucleation process, but rather we introduce bubbles of different initial sizes to imitate the effect of bubbles having nucleated at different times. Thus, our initial conditions (in terms of dimensionless variables) are:

$$
P_{g 1}=P_{g 2}=1 ; \quad c=\Phi ; \quad \boldsymbol{A}=\boldsymbol{I} ; \quad \boldsymbol{u}=\mathbf{0} .
$$

The subscripts 1 refer to quantities in bubble 1 and subscripts 2 refer to those in bubble 2 . The system at $t=0$ is not in equilibrium, because of the different effects of surface tension on bubbles of different sizes.

The evolution of the system is governed by equations (11-15). Since we now have two different bubbles interacting within our periodic element of fluid, we must pay attention to the boundary conditions on both bubbles. The gas conservation equation (15), integrated over time, becomes 


$$
\begin{gathered}
\frac{\left(p_{a}+\left(p_{g 0}-p_{a}\right) P_{g 1}\right)}{p_{g 0}} a_{b 1}=a_{b 1}^{(0)}+12 N \int_{t} \int_{\mathrm{DE}} \nabla c \cdot \boldsymbol{n} \mathrm{d} s \mathrm{~d} t, \\
\frac{\left(p_{a}+\left(p_{g 0}-p_{a}\right) P_{g 2}\right)}{p_{g 0}} a_{b 2}=a_{b 2}^{(0)}+6 N \int_{t} \int_{\mathrm{BC}} \nabla c \cdot \boldsymbol{n} \mathrm{d} s \mathrm{~d} t .
\end{gathered}
$$

where $a_{b 1}$ and $a_{b 2}$ are the dimensionless areas of bubbles 1 and 2 respectively. Since we have used the radius of the larger bubble as our lengthscale, this bubble has initial area $\pi$.

The boundary conditions at the bubble-liquid interfaces become $\nabla c \cdot \boldsymbol{n}=0$ on the straight edges $\mathrm{AB}, \mathrm{CD}$ and $\mathrm{AE}$ of figure 1, and

$$
\begin{aligned}
c & =\Phi \frac{\left(p_{g 0}-p_{a}\right) P_{g 1}+p_{a}}{p_{g 0}} \text { on } \mathrm{DE}, \\
c & =\Phi \frac{\left(p_{g 0}-p_{a}\right) P_{g 2}+p_{a}}{p_{g 0}} \text { on } \mathrm{BC}, \\
-D e P \boldsymbol{n}+(2(1-\beta) \boldsymbol{E}+\beta(\boldsymbol{A}-\boldsymbol{I})) \cdot \boldsymbol{n} & \\
& =-D e P_{g 1} \boldsymbol{n}+\frac{1}{\Gamma} \boldsymbol{n} \nabla \cdot \boldsymbol{n} \text { on } \mathrm{DE} \\
-D e P \boldsymbol{n}+(2(1-\beta) \boldsymbol{E}+\beta(\boldsymbol{A}-\boldsymbol{I})) \cdot \boldsymbol{n} & \\
& =-D e P_{g 2} \boldsymbol{n}+\frac{1}{\Gamma} \boldsymbol{n} \nabla \cdot \boldsymbol{n} \text { on } \mathrm{BC} .
\end{aligned}
$$

Finally, we consider a virtual work argument in which we consider the work done by the fluid stress, bubble gas pressures, ambient pressure and surface tension in changing the bubble areas an infinitesimal amount. This gives the following dimensionless equation relating the expansion rate of the system to the gas pressure differences:

$$
\int_{A_{f}} \boldsymbol{\sigma}: \boldsymbol{E} \mathrm{d} A_{f}=\frac{D e P_{g 1}}{12} \frac{\mathrm{d} a_{b 1}}{\mathrm{~d} t}+\frac{D e P_{g 2}}{6} \frac{\mathrm{d} a_{b 2}}{\mathrm{~d} t}-\frac{1}{\Gamma}\left(\frac{\mathrm{d} C_{1}}{\mathrm{~d} t}+\frac{\mathrm{d} C_{2}}{\mathrm{~d} t}\right),
$$

in which $A_{f}$ is the fluid area, $C_{1}$ is the length of the bubble interface DE and $C_{2}$ the length of the interface $\mathrm{BC}$.

\subsection{Calculation Method and Geometry}

Following the method described in [5], we base our calculations on the original split Eulerian-Lagrangian scheme introduced by Harlen [9]. The equations are solved on triangular finite elements that move and deform with the fluid. At each time step, an estimate of the global expansion rate $\dot{L} / L$ (where $L$ is the linear dimension of our triangular periodic region) is used to solve the mass 
and momentum equations, and repeated iteration using (22) gives the true expansion rate. The gas diffusion is solved using the same finite elements, and the configuration tensor $\boldsymbol{A}$ is then found by solving equation (13) in the Lagrangian frame where the upper convected derivative reduces to an ordinary time derivative. The full details of this calculation are given in [5]. The only differences to the calculation here are the slight modifications required to take account of the presence of the two different bubbles.

In all the results we shall present here, the larger bubble has initial radius 1 (as prescribed by our nondimensionalisation), the smaller bubble has initial radius $1 / 2$, and the initial bubble centre-to-centre distance is 3 . This means that when the more frequent bubble is the larger, the initial area concentration of gas is $\pi / 6 \sqrt{3} \approx 0.3$, and when the less frequent bubble is larger, the initial gas fraction is $\pi / 9 \sqrt{3} \approx 0.2$.

\subsection{Results and Discussion}

For a system identical to this one but with monodisperse bubbles, we found in earlier work $[6,5]$ that the rate of diffusion of gas through the liquid phase and the proportion of the zero-shear-rate viscosity from the polymer, $\beta$, affect the dynamics of the expansion and the shape, but not the final size, of the bubble. The extension of the two-dimensional hexagonal geometry to include bubbles of two different sizes now allows us to investigate the effects of diffusivity and viscoelasticity on the relative sizes of the bubbles as well as the shape of the gas-liquid interface.

When bubbles are identical, surface tension acts to limit the final bubble size by modifying the equilibrium gas pressure and imposes a circular bubbleliquid interface at long times (or circular arcs connected by black films). When bubbles of different sizes are present, the phenomenon of Ostwald ripening occurs. If there were no interaction between bubbles the final gas pressure difference in a circular bubble of dimensionless radius $R$ would be, from (20) or $(21)$,

$$
P_{g \infty} D e=1 /(\Gamma R) .
$$

Thus, small bubbles would have a larger final gas pressure than large bubbles. However, if the gas can diffuse through the liquid then the gas concentration profile becomes uniform throughout the liquid so that all the bubbles must have the same gas pressure. As the concentration of gas in the liquid falls, the pressure in the small bubbles becomes lower than the critical pressure required to maintain their size. Gas now diffuses into the large bubbles from the smaller ones causing them to shrink in size and ultimately disappear.

For large capillary number the timescale associated with the late, surface 
tension driven, phase of the expansion is much larger than the timescales associated with both the gas diffusion and the viscous, or viscoelastic, expansion and so the system expands to a quasi-equilibrium state before Ostwald ripening occurs causing the smaller bubble to shrink to zero. We will pay most attention to this first stage of bubble development, since in a real polymeric foam, the system would be quenched in some way before ripening occurs. Thus henceforth we will only consider the limit of high capillary number $\Gamma \rightarrow \infty$.

We begin by considering the expansion of bubbles in a Newtonian fluid, $\beta=0$, and consider the effects of gas diffusivity. At early times, the bubbles are small and do not feel each other's presence, so we can model them as circular gas regions surrounded by an annular fluid region. The spherical analogy of this model was discussed in detail in [5]; here we simply note that when there is no surface tension (infinite capillary number) the conservation of momentum equation (12) becomes

$$
D e \frac{\dot{a_{b}}}{a_{b}} \frac{X}{X+a_{b}}=P_{g}
$$

where $X$ is the dimensionless liquid area around the bubble in question. It is not obvious a priori how a finite volume of fluid should be partitioned between bubbles of different sizes to determine $X$; we discuss this problem further in section 4.2. Since we are looking at early times, the bubble area, $a_{b}$, increases as

$$
a_{b}=a_{b}^{(0)}\left[1+\frac{1}{D e}\left(1+\frac{a_{b}^{(0)}}{X}\right) t\right]+O\left(t^{2}\right) .
$$

We can see that the initial growth rate is larger for bubbles with a larger value of $a_{b}^{(0)} / X$. Consequently, if we were to assume that each bubble feels all the fluid around it, so that $X$ is equal for all bubbles, we would expect the larger bubbles to expand faster, resulting in a widening of the bubble size distribution as the foam expands. The observations, however, are not quite so straightforward. In figures 2 and 3 we plot the evolution of bubble area ratio (the ratio of the larger bubble area to the smaller) against time for various different values of the diffusivity $N$. In both of these figures (and all the other results we show for a Newtonian fluid) we assume a nominal polymer relaxation time in order to define our dimensionless diffusion parameter and Deborah number. In fact the dynamics are affected not by $N$ and $D e$ in this case, but only by the ratio between them, which is the Péclet number:

$$
P e=\frac{D e}{N}=\frac{R_{0}^{2}\left(p_{g 0}-p_{a}\right)}{\mathcal{D}(\mu+G \tau)} .
$$

In figure 2, which is for the case in which the more frequent bubble, bubble 2 , is the larger, we see that at early times the bubble area ratio increases from 4 , indicating that the bubble area distribution widens as the simple assumption for $X$ predicts. However, in figure 3, in which the less frequent bubble, bubble 


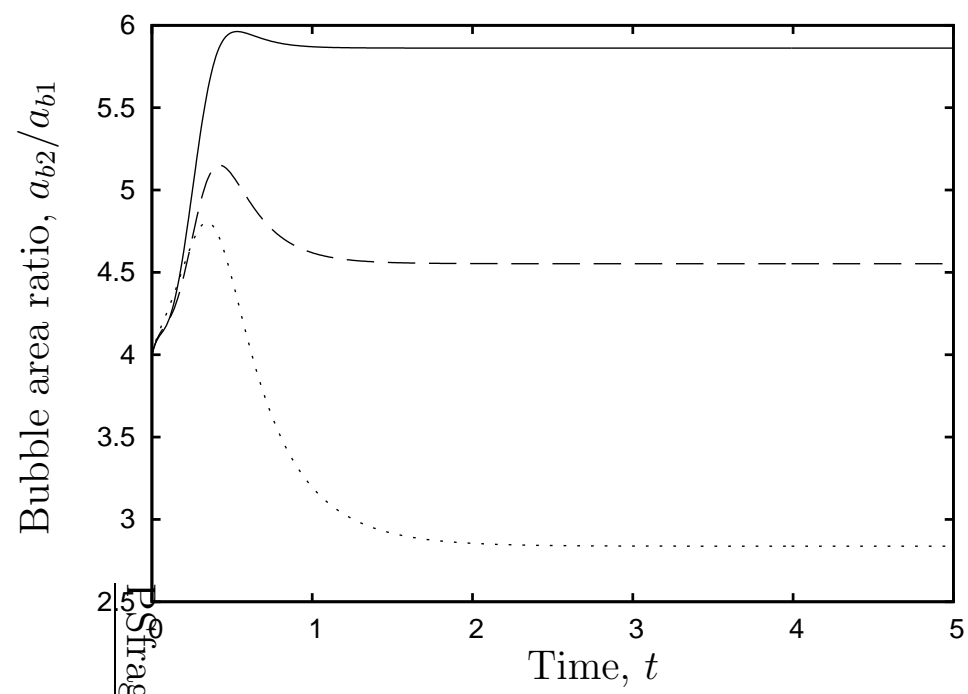

Fig. 2. Evolution of bubble area ratio for bubbles in a ratio of one small to two large bubbles for a Newtonian fluid with $\beta=0, \Gamma=\infty$ and $\Phi=0.32$. The solid line is for fast diffusion $P e=0.9$, the dashed line a moderate value $P e=1.8$ and the dotted line, $P e=9$. The initial bubble area ratio is $a_{b 2}^{(0)} / a_{b 1}^{(0)}=4$.

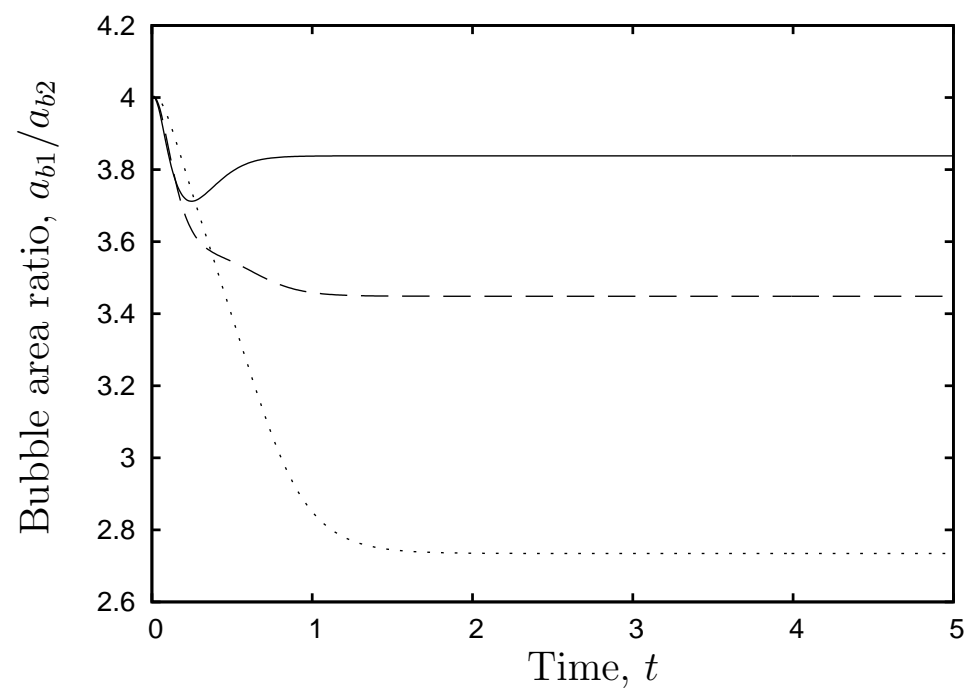

Fig. 3. Evolution of bubble area ratio for bubbles in a ratio of one large to two small bubbles for a Newtonian fluid with $\beta=0, \Gamma=\infty$ and $\Phi=0.32$. The solid line is for fast diffusion $P e=0.9$, the dashed line a moderate value $P e=1.8$ and the dotted line, $P e=9$. The initial bubble area ratio is $a_{b 1}^{(0)} / a_{b 2}^{(0)}=4$.

1 , is the larger, for early times the bubble area ratio decreases, indicating a narrowing of the area distribution. The reason for this discrepancy is that our naïve assumption took no account of the bubble frequency. The more frequent bubble feels a smaller area of liquid, allowing it to have a larger initial growth rate (relative to its size) than the less frequent bubble. Thus the area distribution widens if the more frequent bubble is larger, and narrows if it is smaller. 
After the initial phase, diffusion becomes important. Higher diffusivity causes the big bubbles to grow larger relative to the little ones, essentially because they have a larger surface over which to draw in gas from the liquid. We can see that, in both figures 2 and 3, the final bubble area ratio is larger if diffusivity is high than if it is small.

Once viscoelasticity is introduced into the system, there are three timescales: the polymer relaxation time, the characteristic bubble growth time and the gas diffusion time. These provide the two dimensionless parameters $N$ and $D e$. We begin by considering the case of slow diffusion, $N \ll 1$. The expansion takes place in three stages, just as for an isolated bubble [6]. In the initial expansion phase, the bubbles respond to their initial gas pressure, resisted by the solvent viscosity. The initial growth is faster for viscoelastic fluids as the solvent viscosity is lower (for the same zero-shear-rate viscosity). In the second phase, polymer stresses become important, and the bubble growth rate in a viscoelastic fluid is restricted relative to growth in a Newtonian fluid. The final expansion phase is governed by the slow diffusion timescale: growth is slow and the polymer stresses relax.

In figure 4 we show the bubble areas for the slow-diffusion case, in the configuration of two large to one small bubble. The final bubble sizes are very similar for the different cases, indicating that for slow diffusion, the presence of viscoelasticity has little effect on the final bubble size distribution at these volume fractions.

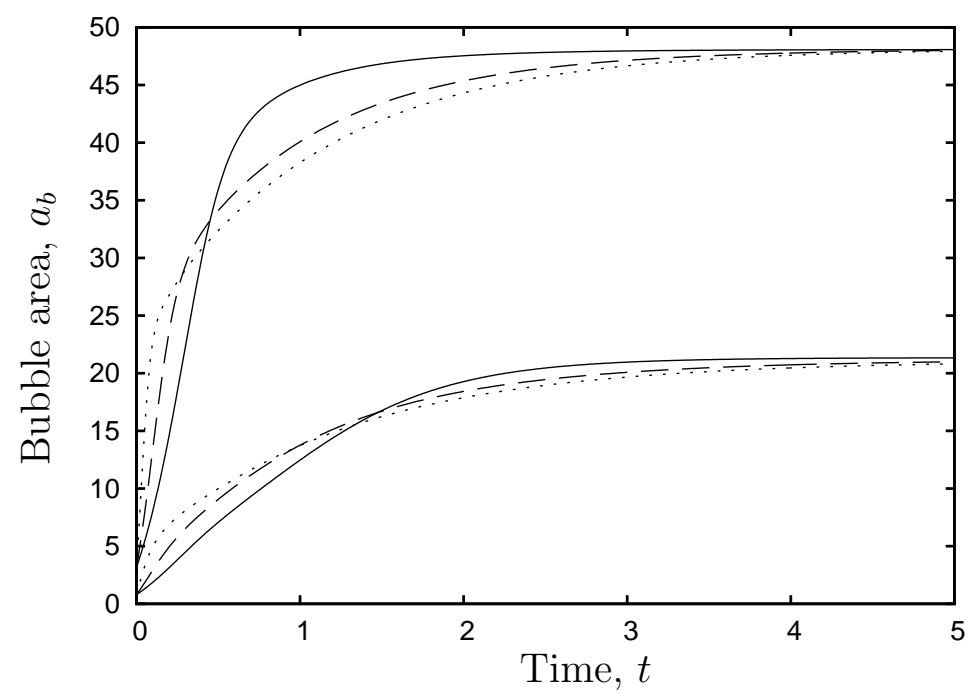

Fig. 4. Growth of the bubbles when diffusion is slow, $N=0.1$. Here there are two large bubbles for each small bubble, $D e=9, \Phi=0.32$ and the capillary number is high, $\Gamma \rightarrow \infty$. The solid line is for a Newtonian fluid $\beta=0$, the dashed line for $\beta=1 / 2$ and the dotted line, $\beta=4 / 5$.

If the bubble volume fraction were much smaller, so that the bubbles had 
little hydrodynamic interaction, we would expect to see a difference in the second phase, when polymer stresses are important. The higher curvature of the smaller bubbles means that the force from the polymer stress in the liquid surrounding the bubbles restricts the size of the small bubble more than the larger ones, resulting in a wider distribution of bubble sizes in viscoelastic liquids compared to Newtonian fluids.

We now look at the case of fast diffusion, $N \gg 1$. The gas available in the liquid is quickly made available to the bubbles resulting in a growth rate which is limited by the fluid stresses on the bubble surface. The expansion is divided into two phases, just as for the case of isolated bubbles [6]: an initial, relatively rapid, viscous phase and a phase controlled by the rate of polymer relaxation. In the latter stage the polymers are extended along the direction of the bubble surfaces, and in particular the windows between neighbouring bubbles, where local stretch has been most rapid, contain very highly stretched polymers and hence very large elastic stresses (much larger than those seen in the slow diffusion case).

We begin with the case of two large bubbles for every small bubble, for which the Newtonian results were given in figure 2. In figure 5 we show the evolution of the bubble area ratio at a diffusivity of $N=10$ for three different values of $\beta$.

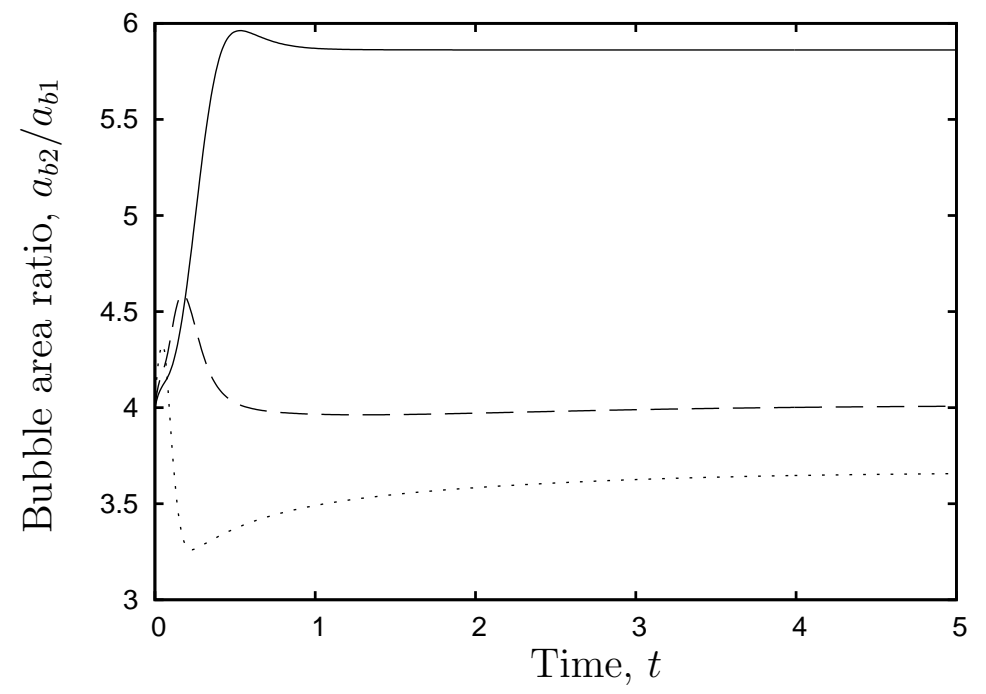

Fig. 5. Evolution of bubble area ratio for bubbles in a ratio of one small to two large bubbles when diffusivity is high, $N=10$. In all curves $D e=9, \Phi=0.32$; the solid line is $\beta=0$, the dashed line $\beta=1 / 2$ and the dotted line $\beta=4 / 5$. The initial bubble area ratio is $a_{b 2}^{(0)} / a_{b 1}^{(0)}=4$.

For the two curves corresponding to viscoelastic fluids with $\beta \neq 0$, the expansion can be split into three phases. In the first, the polymers are unstretched and essentially all our fluids behave as a Newtonian liquid. The effective viscosity at these very early times is not the zero-shear rate viscosity $\mu+G \tau$ 
but the solvent viscosity alone, $\mu$, so for the same value of $D e$ the viscoelastic fluid responds faster. In this very early stage, just as for the Newtonian fluid, the majority bubble expands faster: in this case, as the majority bubble is the larger, the bubble area ratio initially increases.

In the second phase of the expansion, the polymer stresses become important. The highest stresses are in the thinnest window between different bubbles, which is the window between two large bubbles. This elastic stress both restricts the expansion of the large bubbles (shown schematically in figure 6) and also pulls the liquid from the region surrounding the small bubble. This results in the bubble size distribution narrowing as the expansion continues.

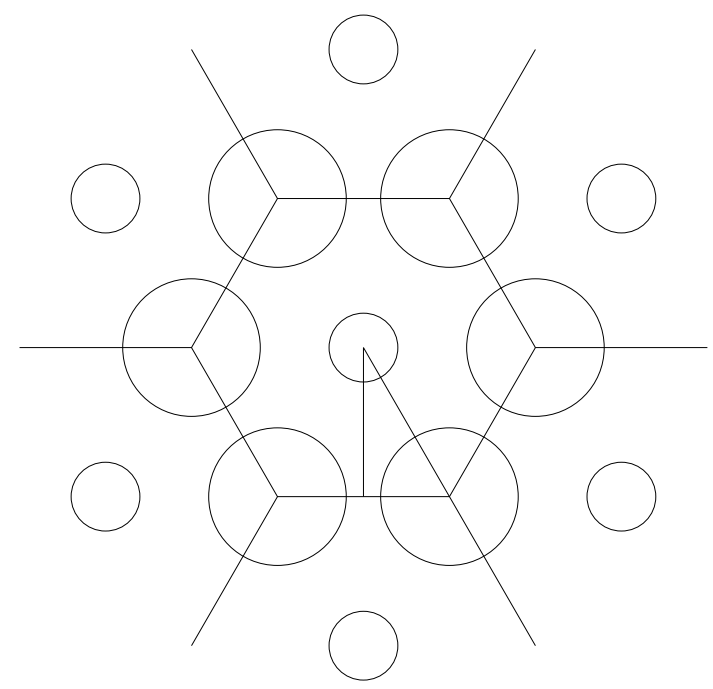

Fig. 6. Schematic diagram showing bubbles in a ratio of two large to one small bubble. Here the large bubbles constrain one another's growth leaving the smaller bubbles in relative isolation.

In the final phase of growth, the supply of gas is running down, the expansion slows, and the polymer stresses relax. As there is little gas remaining in the liquid, diffusion is again the limiting process (even though it is fast) and the large bubbles grow faster due to their larger surface, leading to a slight increase in the bubble area ratio.

The most important of these phases is the second phase, in which elastic stresses govern the growth and narrow the area distribution, and so the final bubble area ratio is smaller for a viscoelastic liquid than a Newtonian in this geometry.

In figure 7 we show the bubble gas pressures for the late stage of this expansion. Here we see that, in the second phase of the expansion, when the polymer stresses build up, the gas pressures in the bubbles in viscoelastic fluids become larger than those in the Newtonian fluid. As the gas supply runs down, towards the end of the expansion, Henry's law guarantees that the gas pressure in the two bubbles will be the same. 


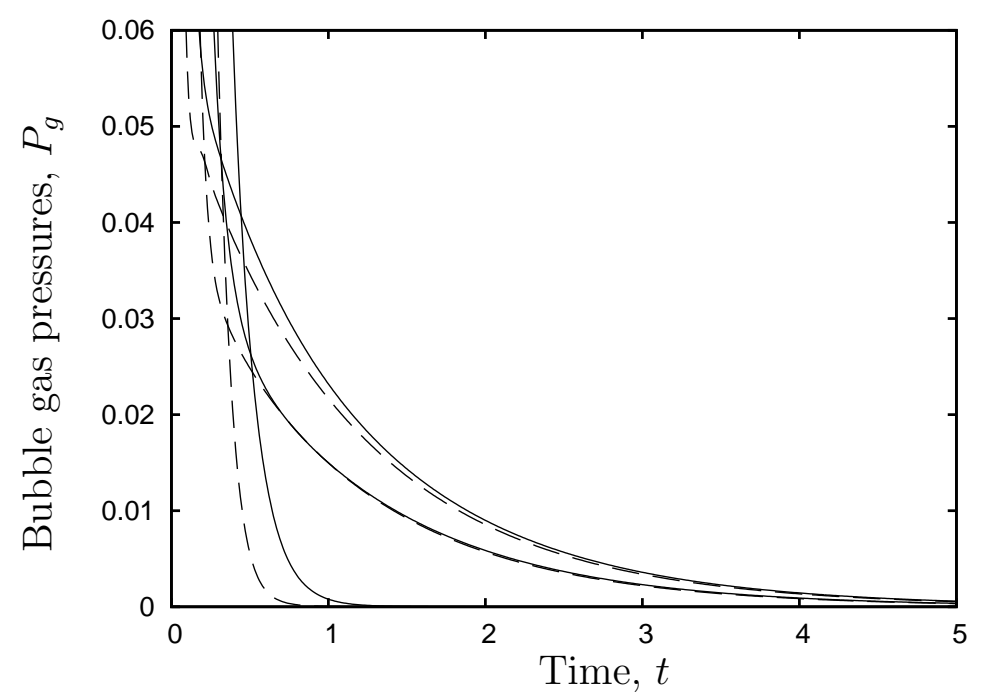

Fig. 7. Comparison of the dimensionless bubble gas pressures $P_{g}$ for bubbles in the ratio of two large to one small bubble between Newtonian liquids and viscoelastic liquids, as described in figure $5(D e=9, \Phi=0.32, N=10, \Gamma \rightarrow \infty)$. Solid lines: small bubble; dashed lines: large bubble. Lower curves: Newtonian liquid; middle curves: viscoelastic liquid, $\beta=1 / 2$; and upper curves: viscoelastic liquid, $\beta=4 / 5$.

We now consider the alternative geometry, in which the smaller bubble is more frequent than the large bubble. In figure 8 we show the evolution of the bubble area ratio at $N=10$ and $D e=9$, for three different values of $\beta$. Here, as for the Newtonian case of figure 3, initially the bubble distribution narrows as the smaller bubbles, being more frequent, are resisted by less fluid. In this early stage, the effective viscosity is $\mu$ rather than the zero-shear-rate viscosity $\mu+G \tau$, so the growth (and the reduction in bubble area ratio) is faster for the viscoelastic fluids, which have a smaller value of $\mu$.

Once elastic stresses become important at later times, the highest stresses are again between the large bubbles. However, each small bubble is now trapped between three large bubbles (illustrated schematically in figure 9), and the effect of these large stresses is to limit the growth of the small bubble, widening the area distribution. In figure 8, we see that the initial Newtonian expansion dominates for these parameter values, resulting in an area distribution which is narrower for viscoelastic fluids than for a Newtonian fluid. However, if we compare instead liquids having the same value of $\mu$, the results are rather different. In figure 10 we compare the evolution of the bubble area ratio for three different scenarios having the same values of $\mu, N$ and $p_{g 0}-p_{a}$, but different values of $\beta$. This means that in our nondimensionalisation the Deborah number is different for the three sets of results.

In figure 10 we see that, if the solvent viscosity $\mu$ is the same for the three fluids, the rôle of the second phase of the expansion, in which the growth of the small bubble is restricted and the size distribution widens, becomes more apparent. Now the early-time behaviour of the three systems is the same, and 


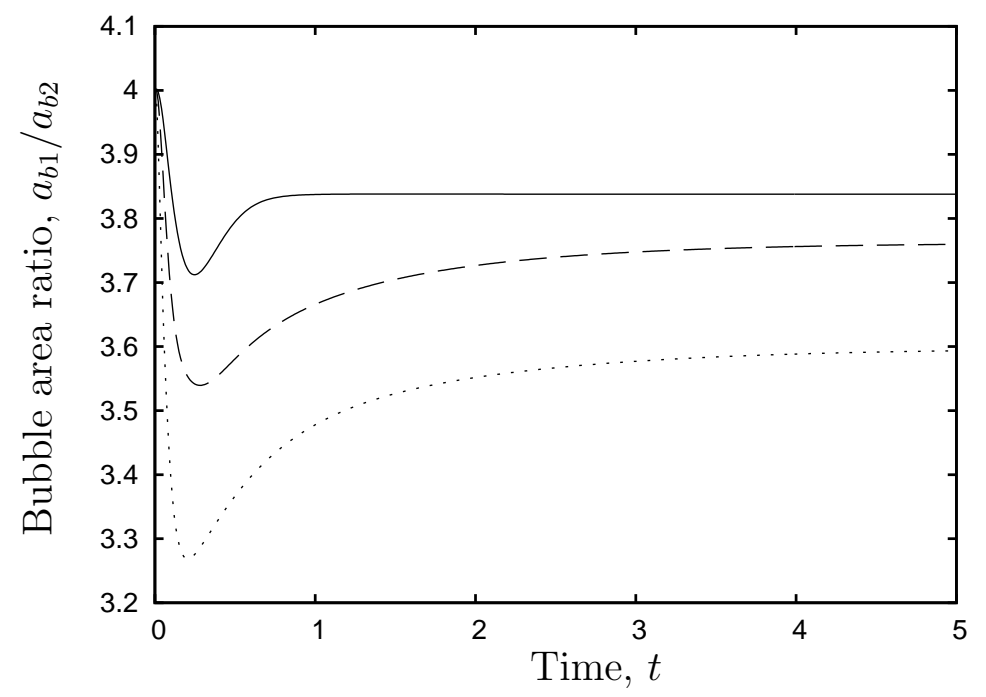

Fig. 8. Evolution of bubble area ratio for bubbles in a ratio of one large to two small bubbles when diffusivity is high, $N=10$. In all curves $D e=9, \Phi=0.32$; the solid line is $\beta=0$, the dashed line $\beta=1 / 2$ and the dotted line $\beta=4 / 5$. The initial bubble area ratio is $a_{b 1}^{(0)} / a_{b 2}^{(0)}=4$.

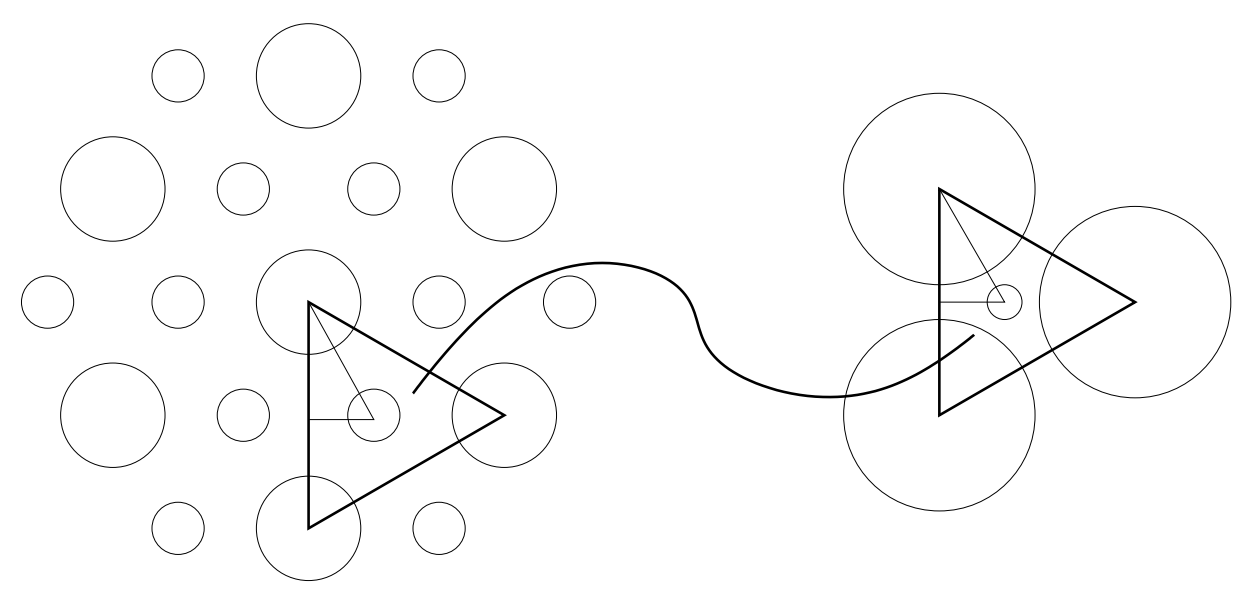

Fig. 9. Schematic diagram showing bubbles in a ratio of one large to two small bubbles. The expanded section illustrates how three large bubbles restrict the growth of a small bubble as time progresses.

the effect of viscoelasticity is to widen the bubble size distribution.

In both of these configurations, the expansion in the viscous phase is much faster than for low diffusivity, resulting in higher polymer stresses. The windows between neighbouring large bubbles are thinner, and those between large and small bubbles are thicker, than in the low-diffusion case.

In summary, when diffusivity is low, we find that the presence of viscoelasticity results in a wider distribution of bubble sizes than would be found with an equivalent Newtonian fluid; and when diffusion is fast, the geometric arrangement of our bubbles determines whether the size distribution is made wider 


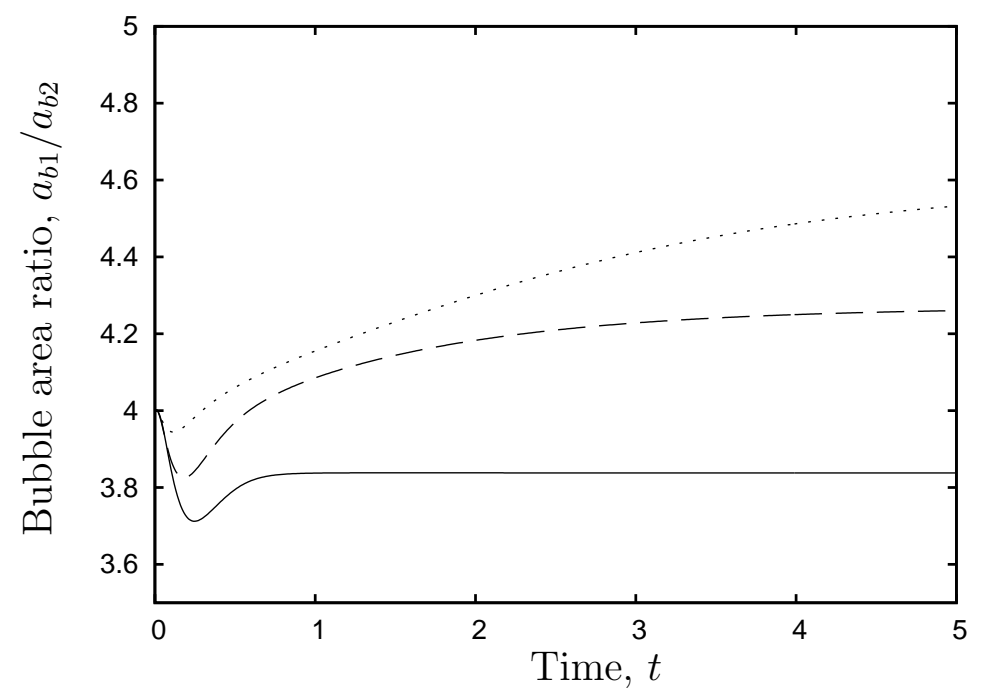

Fig. 10. Evolution of bubble area ratio for bubbles in a ratio of one large to two small bubbles when diffusivity is high, $N=10$. The solvent viscosity $\mu=10^{5} \mathrm{~N} \mathrm{~s} \mathrm{~m}^{-2}$ is the same for all curves, as is the initial pressure difference $p_{g 0}-p_{a}=9 \times 10^{5} \mathrm{~N} \mathrm{~m}^{-2}$. The solid line is $\beta=0, D e=9$; the dashed line is $\beta=1 / 2, D e=4.5$; and the dotted line is $\beta=4 / 5, D e=1.8$. The initial bubble area ratio is $a_{b 1}^{(0)} / a_{b 2}^{(0)}=4$.

or narrower by viscoelastic stresses.

To conclude this section, in figures 11 and 12 we compare snapshots of part of the developing foam in each configuration of bubbles, at a moderate diffusivity value $N=1$ and with viscoelasticity $\beta=1 / 2$. The ratio of initial bubble radii here is 2 (as in all the figures in this section) and the grey scale shows the elastic stress difference in the liquid, plotted as the difference in the eigenvalues of $\boldsymbol{A}$. In either configuration the majority bubble is pulled into a triangular shape.

\section{Foam of isolated spherical bubbles with instant diffusion}

In the previous section we saw that, where diffusion was fast relative to the polymer relaxation time and the viscous growth time, the geometrical constraints imposed by the two-dimensional hexagonal bubble arrangement had significant effects on the bubble dynamics. In order to relax these geometrical constraints, in this section we consider bubbles in three dimensions which are isolated as far as the fluid mechanics of the system is concerned, but which interact through direct competition for a limited supply of available gas.

In order to make analytical progress on this problem, we consider the case where diffusion is instantaneous and so the concentration of gas in the liquid is uniform throughout, and thus by Henry's law all bubbles must have the 


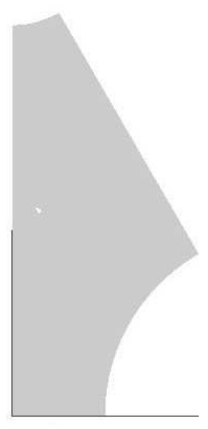

(a) $t=0$.

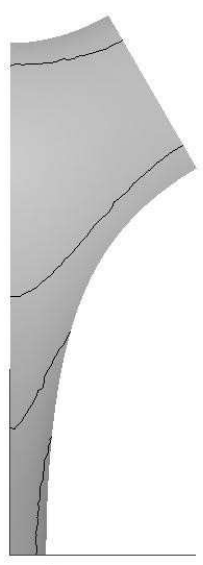

(b) $t=0.2$.

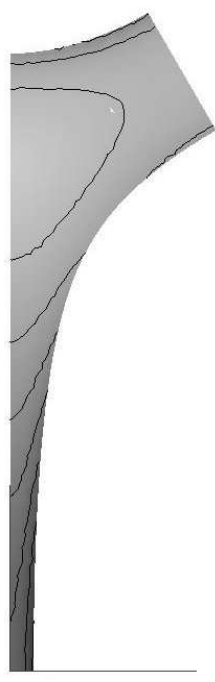

(c) $t=0.4$.

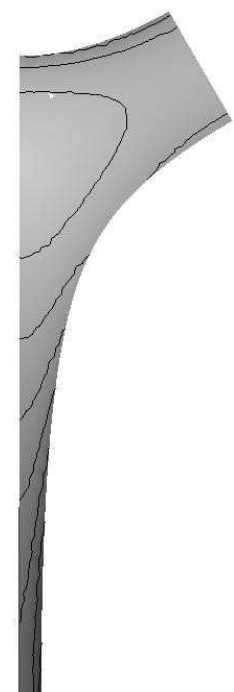

(d) $t=0.5$.

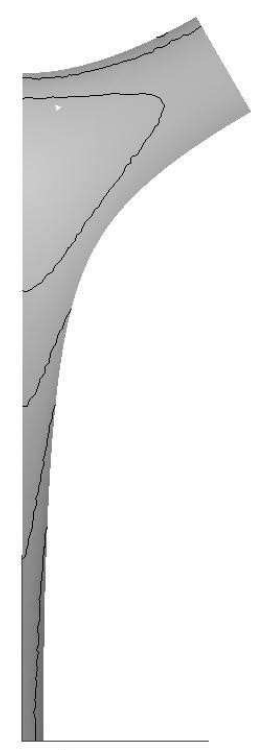

(e) $t=1.0$.

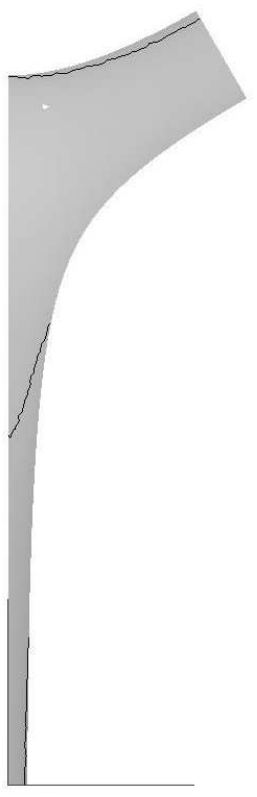

(f) $t=2.0$.

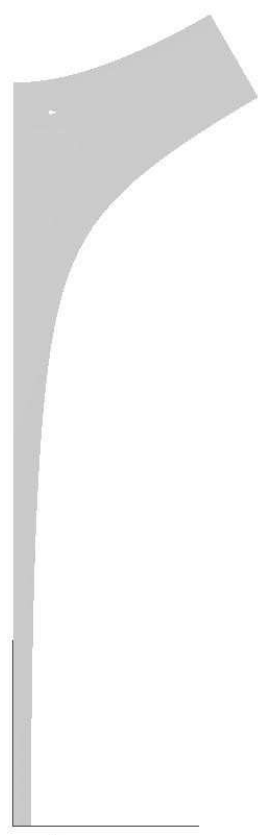

(g) $t=5.0$.

Fig. 11. Snapshots of the developing foam for a configuration of two large to one small bubble. Here $D e=4.5, \beta=0.5, \Phi=0.32, N=1$ and we are in the high-capillary number limit $\Gamma \rightarrow \infty$. The grey scale indicates the magnitude of the difference in the eigenvalues of $\boldsymbol{A}$, and contours are in intervals of 2 . 


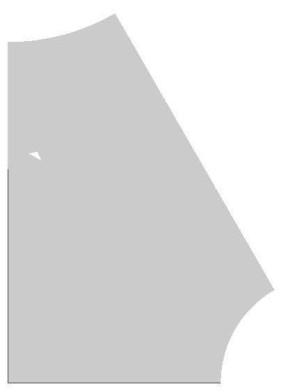

(a) $t=0$.

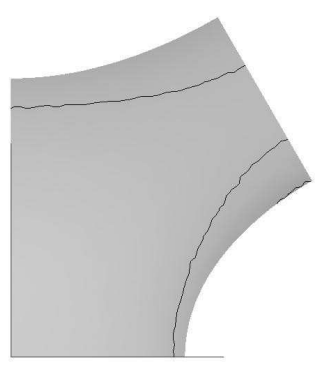

(b) $t=0.2$.

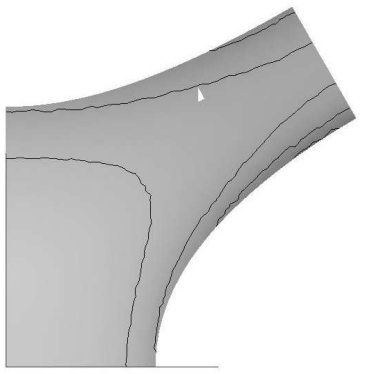

(c) $t=0.4$.

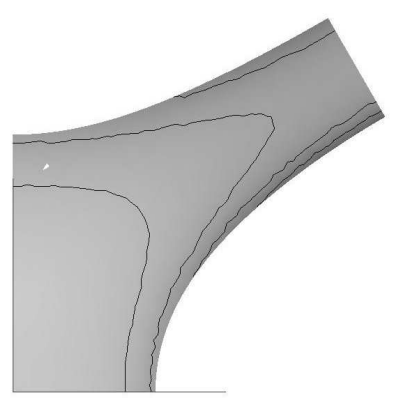

(d) $t=0.5$.

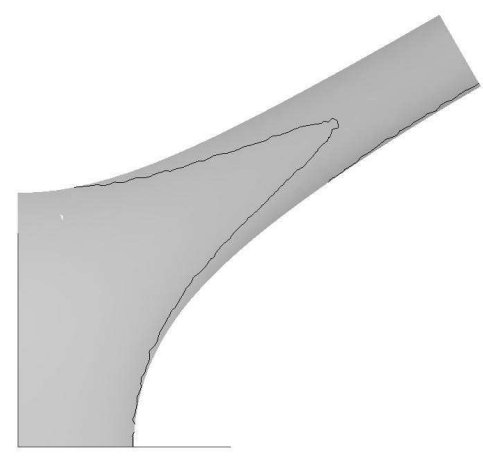

(f) $t=2.0$.

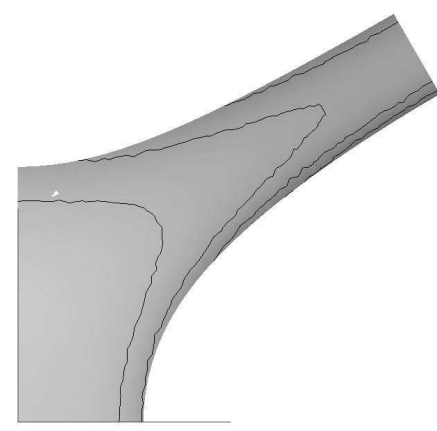

(e) $t=1.0$.

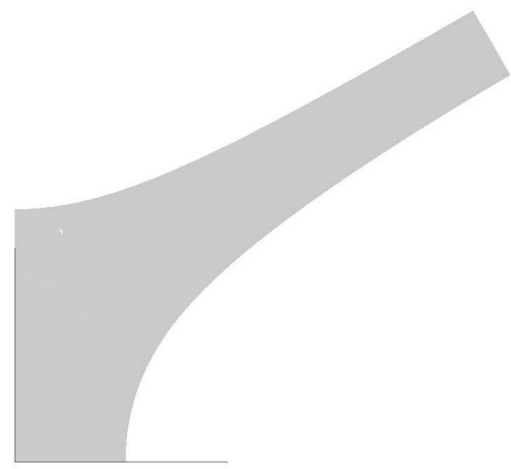

(g) $t=5.0$.

Fig. 12. Snapshots of the developing foam for a configuration of one large to two small bubbles. Here $D e=4.5, \beta=0.5, \Phi=0.32, N=1$ and we are in the high-capillary number limit $\Gamma \rightarrow \infty$. The grey scale indicates the magnitude of the difference in the eigenvalues of $\boldsymbol{A}$, and contours are in intervals of 2 . 
same gas pressure at any instant in time. Furthermore we assume that the bubbles are sufficiently far apart that the bubbles do not interact through fluid motion but only through the concentration of available gas. This simplifies the equations of motion considerably, as the bubbles remain spherical. We will compare the results of this simple approximation to the full two-dimensional simulations of section 3 .

As mentioned in section 3, we will not attempt to model nucleation here. In our model we shall consider two bubbles of differing sizes to represent bubbles that nucleate at different times. The initial bubble volumes are treated as input parameters. Since the bubbles are sufficiently far apart that they do not interact hydrodynamically, we assume that each bubble is working against an infinite fluid domain (although gas conservation will be carried out in a finite domain).

\subsection{Governing Equations}

We are considering here the case in which diffusion of the gas driving the foam expansion is instantaneous, so we cannot use (14) to calculate the supply of gas. The fast diffusion means that the gas concentration, $c$, must be uniform throughout the fluid at all times. Consequently both bubbles, regardless of radius, must have the same gas pressure $p_{g}=c / H$ through Henry's law. We are using dimensional quantities here.

In order to restrict the supply of gas, we assume that both the bubbles are contained in a limited volume of fluid, $4 \pi X / 3$, so that the available gas in the fluid is $4 \pi c X / 3$. Nonetheless, the bubble expansion will be resisted by an infinite volume of fluid.

If we write the volume of each bubble as $4 \pi V_{b i} / 3$ then the gas law gives the gas in a single bubble as $4 p_{g} \pi V_{b i} / 3 R T$, where $R$ is the gas constant and $T$ temperature. The total gas in the bubbles and the fluid is constant in time, and conservation of gas is expressed as

$$
p_{g}\left[V_{b 1}+V_{b 2}+R T H X\right]=p_{g 0}\left[V_{b 1}^{(0)}+V_{b 2}^{(0)}+R T H X\right],
$$

where $V_{b i}^{(0)}$ is the initial value of the volume $V_{b i}$.

Converting to dimensionless variables, we obtain

$$
\left(p_{a}+\left(p_{g 0}-p_{a}\right) P_{g}\right)\left(v_{b 1}+v_{b 2}\right)=p_{g 0}\left(v_{b 1}^{(0)}+v_{b 2}^{(0)}\right)+\Phi\left(p_{g 0}-p_{a}\right)\left(1-P_{g}\right) X,
$$

in which lengths were scaled with $R_{0}$, the initial radius of the larger bubble, as before. We will take bubble 1 to be the larger bubble and note that this gives $v_{b 1}^{(0)}=1$. 
In the neighbourhood of each bubble, the flow is locally radial and spherically symmetric, so the equation of mass conservation (11) gives

$$
u_{r}=\frac{R^{2} \dot{R}}{r^{2}}
$$

where $u_{r}$ is the component of $\boldsymbol{u}$ in the radial direction, $R$ is the radius of the bubble, and $r$ is a general radial coordinate. Due to the spherical symmetry, the angular orientation tensor components $A_{\theta \theta}$ and $A_{\phi \phi}$ are equal, and $p, A_{r r}$ and $A_{\theta \theta}$ depend on $r$ and $t$ only. The radial component of the momentum equation (12) gives

$$
0=-D e \frac{\partial P}{\partial r}+\frac{1}{r^{2}} \frac{\partial}{\partial r}\left(r^{2} \sigma_{r r}\right)-\frac{2 \sigma_{\theta \theta}}{r}
$$

in which $\sigma$ is the Oldroyd-B extra stress:

$$
\boldsymbol{\sigma}=2(1-\beta) \boldsymbol{E}+\beta(\boldsymbol{E}-\boldsymbol{I}) .
$$

Evaluating the derivative of $\sigma_{r r}$ and rearranging allows us to write

$$
D e \frac{\partial P}{\partial r}=\frac{\partial \sigma_{r r}}{\partial r}+\frac{2\left(\sigma_{r r}-\sigma_{\theta \theta}\right)}{r} .
$$

It is convenient to transform from the radial co-ordinate $r$ to a Lagrangian volume co-ordinate $x$ such that $r^{3}=v_{b}+x$ where $(4 / 3) \pi v_{b}$ is the bubble volume. At the bubble surface, $x=0$,

$$
-D e P+\sigma_{r r}=-D e P_{g}+\frac{2}{\Gamma} v_{b}^{-1 / 3}
$$

while as $x \rightarrow \infty, P \rightarrow 0$. Integrating (30) across the fluid layer and using these boundary conditions gives:

$$
0=D e P_{g}+\frac{2}{3} \int_{0}^{\infty} \frac{\sigma_{r r}-\sigma_{\theta \theta}}{x+v_{b}} \mathrm{~d} x-\frac{2}{\Gamma} v_{b}^{-1 / 3} .
$$

In this spherically symmetric flow, the constitutive equation for an Oldroyd B fluid (29) gives

$$
\sigma_{r r}-\sigma_{\theta \theta}=-2(1-\beta) \frac{\dot{v_{b}}}{x+v_{b}}+\beta\left(A_{r r}-A_{\theta \theta}\right)
$$

and so the integrated momentum equation (32) becomes

$$
\frac{4}{3}(1-\beta) \frac{\dot{v_{b}}}{v_{b}}=D e P_{g}+\frac{2}{3} \beta \int_{0}^{\infty} \frac{A_{r r}-A_{\theta \theta}}{x+v_{b}} \mathrm{~d} x-\frac{2}{\Gamma} v_{b}^{-1 / 3} .
$$

Equation (13) gives expressions for $A_{r r}$ and $A_{\theta \theta}$ which can then be subtracted to give an equation for the first normal stretch difference. Due to the coordinate 
transformation the stretch at any position in the fluid can be followed in the Lagrangian frame giving

$$
\begin{gathered}
\frac{\partial A_{r r}}{\partial t}=-\frac{4 \dot{v}_{b}}{3\left(x+v_{b}\right)} A_{r r}-\left(A_{r r}-1\right) \\
\frac{\partial\left(A_{r r}-A_{\theta \theta}\right)}{\partial t}=\frac{2 \dot{v}_{b}}{3\left(x+v_{b}\right)}\left[\left(A_{r r}-A_{\theta \theta}\right)-3 A_{r r}\right]-\left(A_{r r}-A_{\theta \theta}\right) .
\end{gathered}
$$

Initially $A_{r r}=1$ and $A_{r r}-A_{\theta \theta}=0$ everywhere.

The expansion of the bubbles in this section is governed by the momentum equation (33) for each bubble, the evolution equations (34)-(35) for the orientation tensor $\boldsymbol{A}$ around each bubble, and the gas conservation equation (26) globally. Initially we have $P_{g}=1$.

\subsection{Competition between two bubbles}

For a Newtonian fluid in the limit of infinite capillary number the expansion rate, $\dot{v}_{b} / v_{b}$, from equation (33) simplifies to

$$
\frac{4}{3} \frac{\dot{v_{b}}}{v_{b}}=P_{g} D e .
$$

This expansion rate is independent of the size of the bubble, thus the ratio of bubble volumes remains constant in time. In a viscoelastic liquid the increasing polymer stretch difference reduces the growth rate from that of a Newtonian fluid of equal solvent viscosity. However, the smaller bubble is affected to a larger extent than large bubbles due to the $1 /\left(x+v_{b}\right)$ term in the elastic stress in equation (33), resulting in a wider distribution of final bubble volumes. We saw this effect in the hexagonal array at low diffusivities, where the higher surface curvature of the small bubbles made the elastic stresses more restrictive of bubble growth.

Since the total amount of gas in our system is fixed, the sum of the bubble volumes for long times is known before the simulation begins. Thus we can gain most information about the behaviour of the system by looking at the ratio of bubble volumes. In figure 13 we plot the relative final bubble ratio, $\left(v_{b 2} / v_{b 1}\right) /\left(v_{b 2}^{(0)} / v_{b 1}^{(0)}\right)$ as a function of initial volume ratio for two bubbles in a viscoelastic liquid at infinite capillary number. We see that viscoelasticity widens the distribution of bubble sizes compared to a Newtonian liquid, with the widening effect more dramatic for more extreme initial bubble volume ratios. However, at finite capillary number this will only persist provided the system is quenched before surface tension eliminates the smaller bubble through Ostwald ripening. 


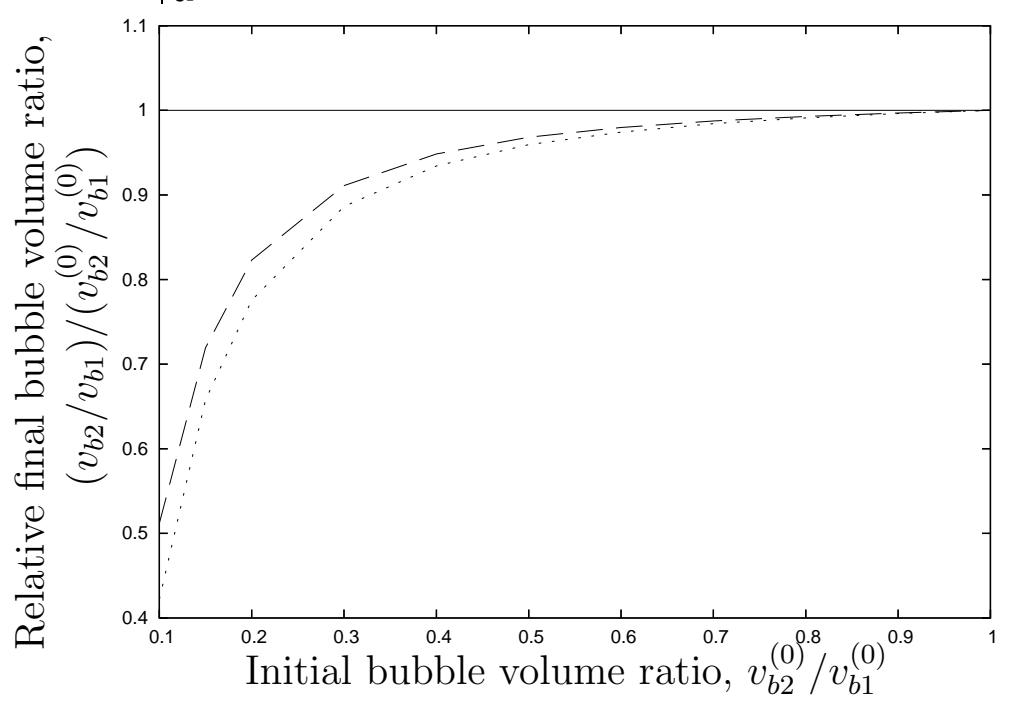

Fig. 13. The effect of viscoelasticity on the final bubble volume ratio as a function of initial volume ratio in the limit of large capillary number. The function shown is the final bubble volume ratio divided by the initial bubble volume ratio. The solvent viscosity $\mu$ and relaxation time $\tau$ are the same for the three curves, and $\Phi=0.32$ throughout. Solid line: Newtonian liquid, $\beta=0, D e=9$ (here the final volume ratio equals the initial volume ratio); dashed line: viscoelastic liquid with $\beta=5 / 6$, $D e=1.5$; dotted line: viscoelastic liquid with $\beta=10 / 11, D e=0.82$.

Finally, in figure 14 we attempt a direct comparison between our two models. We take a high value of the diffusivity $(N=500)$ in the full two-dimensional simulation of the hexagonal array, and make a comparison with the limit of infinite diffusion for perfectly circular bubbles, analogous to the spherical model we have used in this section. Here the bubbles expand in an infinite fluid domain but in the hexagonal array there is a specific quantity of fluid between the bubbles. In order to make a direct comparison in figure 14 we use the standard geometry of section 3 for the case when the larger bubble is less frequent, giving an initial bubble area fraction of $\pi / 9 \sqrt{3}$; when the larger bubble is more frequent, we now use an initial bubble centre-to-centre distance of $\sqrt{15}$ to maintain this initial bubble area fraction. For the spherical model, we need to partition the finite volume of fluid between the two bubbles. In figure 14 we compare the hexagonal simulation for bubbles in both configurations with the circular approximation with fluid partitioned either (a) proportional to the initial bubble areas; or (b) proportional to the initial bubble circumferences (or radii). In the former partitioning, the problem is self-similar and so the bubble area ratio does not change with time.

Since the results of the full two-dimensional simulation depend on the relative number density of large bubbles to small bubbles, we would not expect to see quantitative predictions from these circular approximations: but we can see that partitioning by bubble area gives the nearer prediction, and that the true result for the final bubble areas lies between our two approximations. 


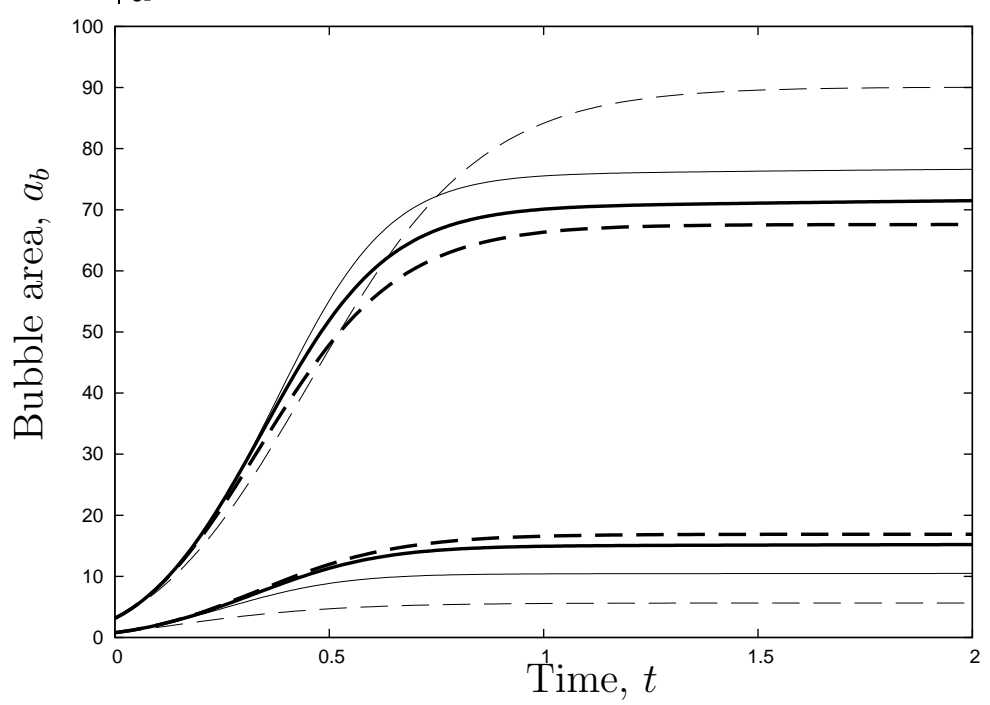

Fig. 14. Comparison, for a Newtonian fluid $\beta=0$, of the full two-dimensional simulation at high diffusivity $(P e=0.018)$ with approximations assuming perfectly circular bubbles and partitioning fluid so that: thick dashed line - fluid area partitioned proportional to the initial bubble area; thin dashed line - fluid area partitioned proportional to the initial bubble circumference. In the $2 \mathrm{D}$ simulations, the thick solid curves are for two small bubbles to one large bubble, and the thin solid curves for two large bubbles to one small bubble. The upper curves are the area of the large bubble, the lower the area of the small bubble. $\Phi=0.32$. Initially the large bubble has area $\pi$ and the small bubble, $\pi / 4$.

\section{Conclusions}

We have used two approximations to model the effects of competition and interaction between bubbles on their growth rates, final sizes and shapes.

Using a full two-dimensional finite-element simulation, we considered a bidisperse hexagonal array of bubbles in which one bubble size occurred twice as frequently as the other. At low rates of diffusion the interfaces between the bubbles remained relatively thick and the bubbles themselves remained approximately circular for much of the expansion process. When viscoelasticity was added to this system, the dynamics of the growth were different but there was little change in the final bubble size distribution. This is in contrast to the case when bubbles are very far apart, in which the higher curvature of the small bubbles means that their growth is inhibited more than for the large bubbles, so the bubble size distribution is widened by the addition of viscoelasticity. This mechanism is essentially the same as that of Ostwald ripening, in which surface tension exerts a stronger effect on smaller bubbles causing them to shrink and eventually disappear.

However, for moderate to high rates of diffusion, when the elastic stresses (rather than available gas) limit the bubble growth, the interfaces between 
neighbouring bubble becomes thin and highly stretched. In this case the geometrical arrangement of bubbles is critical, as the highest stresses occur near the edges of the larger bubbles. If the small bubble is more frequent than the large, each small bubble becomes "trapped" in a triangular region between three large bubbles and its growth is inhibited; on the other hand if the large bubble is the more frequent, the large bubbles inhibit one another's growth and the stresses in the borders between them draw fluid away from the small bubble, enhancing its growth.

As a comparison, we considered a fast-diffusion system in three dimensions in which two bubbles compete directly for the available volume of gas, but each expands in isolation, so that the bubbles remain spherical. We illustrated how, in the same way as for the low-diffusion case of our hexagonal system, viscoelastic stresses have a larger effect on smaller bubbles, widening the final bubble size distribution relative to that for the same bubbles growing in a Newtonian fluid. A direct comparison between the two models shows only qualitative agreement: thus although we cannot use this simpler computation for quantitative prediction, we can have confidence that the conclusions we draw from it are justified.

The systems we have considered here are a first attempt to model polydisperse foaming polymer systems. The assumptions we have made are rather restrictive: in a disordered system we would expect to see a polydisperse (rather than bidisperse) bubble size distribution, and there is the possibility of fast rearrangement events in which inertia may play a part. Nonetheless, we have shown that for a wide range of conditions, viscoelasticity widens the bubble size distribution. Under the conditions where this does not apply, for fast diffusion with closely-packed bubbles, we have shown that the critical factor in determining the bubble growth rate is the regions of high polymer stress between the largest bubbles in the system.

\section{Acknowledgements}

Sally Everitt would like to acknowledge the EPSRC and Huntsman Polyurethanes (an international business of Huntsman International LLC) for their financial support. We would also like to thank Tim Nicholson for the use of his flow visualisation software, FlowDis, and Don Jones for many useful discussions. 


\section{References}

[1] M Amon and C Denson. A study of the dynamics of foam growth: Analysis of the growth of closely spaced spherical bubbles. Polymer Engg Sci., 24:1026-1034, 1984.

[2] A Arefmanesh and S G Advani. Diffusion induced growth of a gas bubble in a viscoelastic fluid. Rheol. Acta, 30:274-283, 1991.

[3] A Arefmanesh and S G Advani. Non-isothermal bubble growth in polymeric foams. Polymer Engg Sci., 35:252-260, 1995.

[4] A Arefmanesh, S G Advani, and E E Michaelides. An accurate numerical solution for mass diffusion induced bubble growth in viscous liquids containing limited dissolved gas. Int. J. Heat Mass Trans, 35:1711-1722, 1992.

[5] S L Everitt, O G Harlen, and H J Wilson. Bubble growth in a twodimensional viscoelastic foam. J. Non-Newt. Fluid Mech., 2006. (In press).

[6] S L Everitt, O G Harlen, H J Wilson, and D J Read. Bubble dynamics in reacting and non-reacting polymer foams. J. Non-Newt. Fluid Mech., 114:83-107, 2003.

[7] M Favelukis. Dynamics of foam growth: Bubble growth in a limited amount of liquid. Polymer Engg Sci., 44:1900-1906, 2004.

[8] C J Han and H J Yoo. Studies on structural foam filling. IV. Bubble growth during mould filling. Polymer Engg Sci., 21:518-533, 1981.

[9] O G Harlen, J M Rallison, and P Szabo. A split Lagrangian-Eulerian method for simulating transient viscoelastic flows. J. Non-Newt. Fluid Mech., 60:82-104, 1995.

[10] A W Hodgson. Homogeneous nucleation. Adv. Coll. Int. Sci, 21:303-327, 1984.

[11] C Pozrikidis. Expansion of a two-dimensional foam. Engineering Analysis with Boundary Elements, 26:495-504, 2002.

[12] N S Ramesh and N Malwitz. A non-isothermal model to study the influence of blowing agent concentration on polymer viscosity and gas diffusivity in thermoplastic foam extrusion. J. Cell. Plas., 35:199-209, 1999.

[13] N S Ramesh, D H Rasmussen, and G A Campbell. Numerical and experimental studies of bubble growth during the microcellular foaming process. Polymer Engg Sci., 31:1657-1664, 1991.

[14] E Ruckenstein and B Nowakowski. A kinetic theory of nucleation in liquids. J. Coll. Int. Sci., 137(2):583-592, 1990.

[15] L W Schwartz and R V Roy. Interim report: Theoretical and numerical modelling of an expanding foam. Technical report, University of Delaware, 2000.

[16] M A Shafi and R W Flumerfelt. Initial bubble growth in polymer foam processes. Chem. Engg Sci., 52:627-633, 1997.

[17] M A Shafi, K Joshi, and R W Flumerfelt. Bubble size distribution in freely expanded polymer foams. Chem. Engg Sci., 52:635-644, 1997. 
[18] V K Shen and P G Debenedetti. A kinetic theory of homogeneous bubble nucleation. J. Chem. Phys., 118(2):768-783, 2003.

[19] J R Street. The rheology of phase growth in elastic liquids. Trans. Soc. Rheol., 12:103-131, 1968.

[20] I Tanasawa and W Yang. Dynamic behavior of a gas bubble in viscoelastic fluids. J. Appl. Phys., 41:4526-4531, 1970.

[21] R Ting. Viscoelastic effect of polymers on single bubble dynamics. A.I.Ch.E. J., 21:810-813, 1975. 